\title{
Roamer, un robot en el aula de Educación Infantil para el desarrollo de nociones espaciales básicas
}

\author{
Noelia Bizarro Torres ${ }^{1}$, Ricardo Luengo González ${ }^{1}$, José Luís Carvalho ${ }^{1}$ \\ noebizarro87@gmail.com, rluengo@unex.es, jltc@unex.es \\ ${ }^{1}$ Universidad de Extremadura, Facultad de Educación-Grupo de Investigación Ciberdidact, Departamento de \\ Didáctica de las Ciencias Experimentales y Matemáticas. Avda de Elvas s/n, 06006, España.
}

DOI: 10.17013/risti.28.14-28

\begin{abstract}
Resumen: El tratamiento de Robótica educativa en Educación Infantil lleva implícita la iniciación del pensamiento computacional y el apoyo al desarrollo de nociones espaciales básicas (delante, detrás, arriba, abajo, derecha e izquierda). Por ello, a través de la presente propuesta didáctica desarrollada en el aula se pretende conocer las diferencias sobre la adquisición de estos conceptos espaciales básicos antes y después de la intervención con el Robot Roamer. Se ha diseñado una investigación de enfoque cualitativo utilizando como instrumentos de investigación una prueba vivencial y una prueba de dibujo que han sido adaptadas a las características evolutivas de los niños de Educación Infantil. Para el apoyo al análisis cualitativo de datos se ha utilizado el software webQDA. A modo de conclusión, el trabajo con robótica en Educación Infantil mejora la adquisición de conceptos espaciales básicos. Sin embargo, los conceptos derecha e izquierda siguen siendo los más complicados de adquirir y asimilar.
\end{abstract}

Palabras-clave: Robótica educativa, Educación Infantil, Nociones espaciales básicas, análisis cualitativo, webQDA.

\section{Roamer, a robot in the classroom of Early Childhood Education for the development of basic spatial notions}

\begin{abstract}
The treatment of educational robotics in Early Childhood Education implicitly involves the initiation of computational thinking and support for the development of basic spatial notions (front, back, robot, right, left and right). Therefore, through the present didactic proposal developed in the classroom, it is intended to know the differences in the acquisition of these basic spatial concepts before and after the intervention with Roamer Robot. An investigation of qualitative approach has been designed to use as experimental instruments an experiential test and a drawing test that have been adapted to the evolutive characteristics of children in Early Childhood Education. To support the qualitative analysis of the data, the webQDA software was used. By way of conclusion, the work with robotics in Early Childhood Education improves the acquisition of basic spatial concepts. However, the concepts of the right and the left remain the most complicated to acquire and assimilate.
\end{abstract}

Keywords: Educational robotics; children's education, Spatial notions, webQDA. 


\section{Introducción}

El presente trabajo de investigación resulta de la realización de un estudio piloto previo al proyecto de tesis doctoral "La robótica educativa como soporte al desarrollo de nociones espaciales básicas en Educación Infantil" que se está llevando a cabo en el Departamento de Didáctica de las Ciencias Experimentales y Matemáticas de la Universidad de Extremadura (España). En este estudio piloto nos centramos en la aplicación y el tratamiento de datos cualitativos a través del software webQDA (Ferrão, Marques y Moreira, 2013).

Presentamos una propuesta de intervención educativa para el desarrollo de nociones espaciales básicas en alumnos de Educación Infantil a través de la utilización y programación robótica, concretamente de la utilización del Robot Roamer (Valiant Tecnhonology) en el aula. Para ello, hemos tenido presente todos los aspectos que influyen en el proceso de enseñanza-aprendizaje y que se plasma a través del trabajo metodológico de aula basada en la EMO - Experimentación, manipulación y observación (Bizarro, Luengo y Carvalho, 2018).

El proceso de desarrollo de las nociones básicas lleva implícito el trabajo a través de una serie de fases paralelas que se encuentran relacionadas con las necesidades psicoevolutivas de los alumnos de estas edades. Así, se ha trabajado el desarrollo del propio esquema corporal y la proyección sobre objetos ajenos a nuestro cuerpo, en esta situación el objeto fue el robot Roamer.

La obtención de información se ha llevado a cabo una recogida de datos antes y después de la intervención práctica con Roamer mediante una prueba gráfica y una prueba vivencial que se analizó con el apoyo del software WebQDA. A través de este proceso de recogida, tratamiento y análisis de datos comprobamos si existen diferencias significativas al trabajar en el aula con una metodología activa basada en la programación Robótica.

Los resultados obtenidos nos permiten a los docentes tomar decisiones sobre la mejor opción metodológica y las herramientas a utilizar en el trabajo diario en el aula, para así llevar a cabo una intervención educativa acorde con las necesidades de los alumnos, su contexto y las demandas sociales en las que nos encontramos inmersos.

\section{Planteamiento del problema y contexto.}

La sociedad en la que nos encontramos inmersos demanda profesiones relacionadas con los avances tecnológicos y su aplicación en el mundo industrial. Por ello, es necesario tratar este punto de demanda desde la formación en los primeros años, es decir, desde la Educación Infantil. Se trata de una nueva forma de alfabetización y comunicación con los demás y estrechamente relacionada con las habilidades tradicionales de la lectura, la escritura y las matemáticas.

Desde edades tempranas debemos preparar a nuestros alumnos para nuevas profesiones que actualmente no conocemos pero que seguramente estarán íntimamente ligadas con la robótica y sus potencialidades. Es necesario favorecer el desarrollo de todas y cada una de sus capacidades a través de un recurso motivador, globalizador y con múltiples potencialidades educativas que le permita desarrollar su vida laboral y profesional. 
Teniendo en cuenta lo anteriormente expuesto, seguimos la visión de Papert que expone que "son los niños los que tienen que educar a los ordenadores no los ordenadores los que tienen que educar a los niños" (Papert, 1980, citado por Blikstein, 2013). Por tanto, la robótica no deja de ser una herramienta más en el ámbito educativo, es un mero recurso que va a ayudar a conseguir los objetivos propuestos y que se encuentra estrechamente ligada con las necesidades demandas por la alfabetización digital.

Además, el trabajo con robótica en el aula permite llevar a cabo un enfoque globalizador, ya que se trata de un recurso que se puede adaptar a muchos tipos de contenidos y materias. Sin embargo, en el trabajo de investigación que nos concierne, cabe destacar un ámbito del desarrollo estrechamente ligado con el trabajo de la robótica educativa, el del desarrollo espacial del niño. La programación secuenciada de órdenes, la estructuración de las mismas y el control de un objeto externo a sí mismo como es el robot, dentro de un espacio determinado, puede ayudar al niño a comprender, afianzar y desarrollar su orientación espacial de una manera más positiva y funcional.

Teniendo en cuenta todos estos aspectos, el objetivo principal de la investigación consiste en analizar si el trabajo en el aula con robótica educativa mejora la orientación espacial del alumno de Educación Infantil en cuanto a su propio cuerpo y a los objetos externos. A través de la exploración de este objetivo se pretende dar respuesta a la siguiente pregunta de investigación ¿Mejoran los alumnos de Infantil la adquisición de conceptos espaciales básicos con la utilización de robótica educativa en el aula?

\subsection{El pensamiento computacional como soporte para el desarrollo cognitivo en Educación Infantil.}

El niño de Educación Infantil se encuentra en el estadío preoperacional (2-7 años) según la teoría de desarrollo de Piaget (Felman, 2007). En esta etapa el niño desarrolla la función simbólica para actuar y comprender el mundo que le rodea, sin embargo, según este autor, no es capaz de desarrollar procesos mentales organizados, formales y lógicos. Por ello, es fundamental trabajar la programación robótica en el aula de Educación Infantil, ya que ayudará al niño a estructurar secuencias y procesos mentales con una lógica funcional y significativa. Este trabajo, que implica resolver problemas, diseñar sistemas y comprender el comportamiento humano, haciendo uso de los conceptos fundamentales de la informática, es también defendido por Wing (2006) en el ámbito del desarrollo de um pensamiento computacional.

Para trabajar el pensamiento computacional en el aula infantil es preciso llevar a cabo un proceso de resolución de problemas que ayudan al niño a estructurar su pensamiento y desarrollar un razonamiento lógico. Esta misma competencia se puede aplicar a cualquier área y etapa educativa, aunque en la etapa de Educación Infantil cobra mayor relevancia, ya que a través del desarrollo de estas estrategias se trabajan aspectos cognitivos que pueden ayudar a superar las limitaciones del pensamiento preoperacional: centración, egocentrismo, irreversibilidad, comprensión incompleta de la trasformación.

Las necesidades y características del alumnado de estas edades hacen necesaria la continua motivación para conseguir un aprendizaje funcional y significativo en el que el alumno es el principal agente de su propio aprendizaje. De esta manera el alumno puede extrapolar los contenidos trabajados en el aula a su mundo inmediato y así 
mantendremos su motivación constante ya que encontrará sentido a lo aprendido en el aula. Por ello, a través del trabajo con robótica en el aula mediante la metodología EMO damos respuesta al principio de actividad y juego, por el que el alumno satisface su curiosidad realizando todo tipo de actividades de observación y exploración a través de las cuales interioriza e incorpora contenidos a su estructura cognitiva.

Otra de las características del pensamiento del niño de Educación Infantil que le impide desarrollar los contenidos en toda su totalidad es el principio de irreversibilidad, por el que el alumno es incapaz de volver al inicio de un razonamiento o percibirlo desde algún cambio espacial. Por ello se partirá del desarrollo de las nociones espaciales de sus propios ejes corporales, para posteriormente extrapolarlo a objetos de referencia que cambian su posición, como el robot Roamer. Este proceso y herramienta ayudará al alumno a percibir e interpretar su realidad más inmediata desde una perspectiva más funcional.

\subsection{Robótica educativa en el aula.}

El trabajo con robótica en Educación Infantil puede ayudar al desarrollo de nociones básicas espaciales referentes al propio cuerpo del niño y a objetos de su entorno. La estructuración de la noción de espacio cobra fuerza a medida que el niño desarrolla el control dinámico y coordinación de su propio cuerpo, además de la toma de conciencia sobre objetos externos a él.

Manuel Valencia, director de Argan Bot en una entrevista al periódico "El español" (2016) defiende que "con la programación conseguimos estructurar la cabeza para que se resuelvan los problemas de manera más lógica”. De esta manera, cuando el niño se enfrenta a un problema tiene que buscar en su estructura cognitiva para analizar la situación y resolver el problema, hitos" que el niño posee en torno a los cuales establecerá "rutas" para crear el procedimiento de trabajo, y posteriormente elegir el más idóneo para la resolución del problema, así lo expone la Teoría de los Conceptos Nucleares (Casas y Luengo, 2011).

Teniendo en cuenta lo anteriormente expuesto, se pueden nombrar algunos aspectos que justifican nuestro proyecto de investigación.

- Es de gran importancia analizar el desarrollo del conocimiento y el control del propio cuerpo referente a la orientación espacial de los niños de Educación Infantil utilizando programación robótica a través de Roamer.

- Analizar y observar si existen diferencias en los resultados extraídos antes y después de la intervención educativa para comprobar si existen diferencias significativas que nos permitan tomar decisiones para mejorar el proceso de enseñanza-aprendizaje.

- Cabe destacar la contextualización legislativa de la utilización de robótica dentro del aula. Para ello, Roamer se integrará como una herramienta que ayudará a potenciar en el alumno el desarrollo espacial como aspecto principal y otros contenidos secundarios relacionados con todas las áreas del currículum oficial de Educación Infantil en nuestra Comunidad Autónoma de Extremadura, Decreto 4/2008 de 11 de Enero, por el que se establece el currículo de Educación Infantil para la Comunidad Autónoma de Extremadura. 
- A través del trabajo en el aula se facilita a los alumnos un enfoque globalizado (Alsina, 2012) como principio esencial para la práctica educativa en Educación Infantil. Así a través de la robótica educativa se trabajan contenidos de diversa índole: matemáticos, lenguaje, observación del entorno, socialización, etc.

- Además, cabe destacar el principio de juego y motivación que proporciona el trabajo con robótica educativa dentro del aula. Es un aspecto íntimamente ligado a las necesidades de aprendizaje de los niños de estas edades. Así se parte siempre de los centros de intereses de los alumnos.

\subsection{Proceso de evaluación en educación infantil.}

La evaluación del proceso de aprendizaje es fundamental, ya que ofrece datos acerca de desarrollo y adquisición de nuevos conocimientos. Se destaca la siguiente definición de evaluación (López-Pastor, 2017) “es aquella actividad de aprendizaje que se evalúa para comprobar los conocimientos, capacidades y competencias que se van adquiriendo, así como los requisitos y condiciones que debe cumplir". Utilizar un instrumento y una herramienta adecuada para el análisis de datos es muy importante. En la etapa en la que se desarrolla el presente estudio, Educación Infantil, se debe tener muy presente las características de los alumnos para desarrollar la actividad de evaluación.

Además hay que tener en cuenta que la evaluación también forma parte del proceso de enseñanza y aprendizaje y es necesario plantearla como una experiencia formativa. Así, un proceso de evaluación vivencial favorece la implicación del niño y es coherente con la metodología de trabajo empleada en la etapa de Educación Infantil.

En un proceso de investigación la mejor técnica de recogida de datos para una evaluación vivencial es la filmación de imágenes y vídeos sobre la ejecución de la actividad por parte de los alumnos. Así se puede analizar detenidamente los logros conseguidos y los errores cometidos, tanto por los alumnos como por el docente. De esta manera, se obtiene información práctica sobre los aspectos positivos que deben mantenerse y los aspectos negativos que deben reforzarse o modificarse.

\subsection{Tratamiento de datos cualitativos: Software WebQDA.}

El software WebQDA, elegido para apoyar el tratamiento y análisis de datos, permite realizar todas las tareas que conlleva el tratamiento de datos en nuestra investigación: organización, categorización y análisis de los mismos (Costa, Faria y Reis, 2016).

webQDA es un software que ha sido creado por la empresa Micro IO y Ludomedia en colaboración con la Universidad de Aveiro (Neri de Souza, Costa, Moreira, Neri de Sousa, 2013). La versión en castellano fue desarrollada con el apoyo del grupo de investigación CiberDidact de la Universidad de Extremadura (España). Se trata de un software específico destinado a la investigación cualitativa en general que possibilita el análisis de datos cualitativos (textos, gráficos, imágenes, vídeos). Además es una herramienta que permite editar, almacenar y organizar documentos, donde se puede crear categorías, codificar, controlar, filtrar, investigar y consultar datos de investigación.

El principal motivo de la elección del software WebQDA para el tratamiento de datos es que permite la incorporación de fuentes de diversa índole: textuales, gráficas, sonoras, etc. 
Al trabajar con alumnos de Educación Infantil, las pruebas diseñadas son muy gráficas y este software permite trabajar con ellas de manera muy fácil y organizada. Además, cabe resaltar la facilidad de organización de los datos de investigación a través de sistemas de categorías. El hecho de poder compartir el proyecto con otros investigadores resulta muy útil para potenciar el trabajo colaborativo (Costa y Reis, 2017).

\section{Metodología}

En referencia al objetivo planteado y la pregunta de investigación, en este estudio se utiliza el método cualitativo basado en instrumentos de recogida de datos por observación directa del profesor y por análisis a través del software webQDA.

\subsection{Muestra}

La selección de la muestra fue obtenida por un muestreo no probabilístico por conveniencia. Los criterios de selección que se tuvieron en cuenta para la investigación fueron los siguientes:

- 1 grupo de alumnos escolarizados en 5 años de Educación Infantil.

- Los alumnos seleccionados deben tener desarrolladas una serie de contenidos básicos conocer números, algunas letras, colores, etc.

- Los alumnos deberán asistir con continuidad al colegio para llevar a cabo una investigación continúa.

Se trató de encontrar una muestra, que cumpliendo los anteriores criterios, fuera accesible para la investigadora, ya que es tutora del grupo. La muestra elegida se ha determinado a partir de los alumnos de Educación Infantil de 5 años del CEIP Ntra. Sra. de Chandavila de localidad de La Codosera (comunidad de Extremadura - España). Se trata de un grupo de 7 alumnos con características y motivaciones diferentes pero con un buen nivel de desarrollo cognitivo. Cabe destacar la participación en el grupo de un niño con Necesidades Educativas Especiales.

De los 7 participantes que asisten habitualmente al colegio, todos participarán activamente en la investigación ya que sus padres han expresado su previa autorización por escrito. Es un grupo muy pequeño que permite un tratamiento muy individualizado.

\subsection{Fases y temporalización}

En referencia a las fases de la investigación, el estudio comenzó con una fase inicial de recogida de datos y diseño de la investigación. La intervención práctica ha tenido una duración de 3 meses desde finales de Noviembre hasta principios de Febrero. Posteriormente a esta intervención práctica se llevó a cabo la fase final de tratamiento de datos. Debido a las características de la metodología de trabajo empleada en Educación Infantil, delimitar la temporalización en número de sesiones específicas es complicado, ya que el trabajo se realiza desde una perspectiva globalizadora donde los contenidos se encuentran interrelacionados constantemente. Por esta característica se define el siguiente plan de trabajo: 


\begin{tabular}{|c|c|c|}
\hline Fases & Número de sesiones & Descripción \\
\hline Evaluación inicial & 2 & Dibujo $^{1}$ y Evaluación Vivencial ${ }^{1}$ \\
\hline $\begin{array}{l}\text { Actividades } \\
\text { prácticas } \\
\text { con Robot } \\
\text { Roamer y de } \\
\text { Psicomotricidad }\end{array}$ & $\begin{array}{l}34 \text { Sesiones concretas y Sesiones } \\
\text { puntuales contextualizadas en los } \\
\text { contenidos y necesidades del grupo. }\end{array}$ & $\begin{array}{l}\text { Actividades combinadas de robótica educativa } \\
\text { y psicomotricidad y contextualizadas en } \\
\text { el aula, por ejemplo. En la asamblea diari } \\
\text { el protagonista del día resolverá un reto } \\
\text { propuesto: dirigir a Roamer hacía la letra "L". }\end{array}$ \\
\hline Evaluación final & 2 & Dibujo $^{2}$ y Evaluación Vivencial ${ }^{2}$ \\
\hline
\end{tabular}

Tabla 1 - Temporalización de la investigación.

\subsection{Instrumentos utilizados}

Los instrumentos de obtención de datos que se han utilizado han sido seleccionados y diseñados teniendo en cuenta las características de los participantes y de la propia investigación. A continuación se describe cada instrumento seleccionado, su justificación y proceso de aplicación en nuestra investigación.

\subsubsection{Evaluación Vivencial.}

Esta actividad consiste en que el alumno identifique una parte determinada del cuerpo de su compañero referente a los conceptos en estudio: delante-detrás; arriba-abajo; derecha-izquierda. Esta identificación se realizó tendiendo presente las posiciones corporales más habituales: de frente, de espaldas y tumbado. Uno de los alumnos hizo la función de maniquí (no podía moverse ni decir nada a su compañero), que fue cambiando para permitir la participación de todos. Otro compañero colocaba pegatinas de un color determinado en la parte del cuerpo del maniquí que indicaba el docente.

Esta evaluación vivencial se llevó a cabo para conocer si el alumno es capaz de diferenciar los conceptos que se van a trabajar en un cuerpo ajeno al propio. Además, se cambia la posición del cuerpo del maniquí para comprobar esta misma adquisición en diferentes ejes de referencia: de frente, de espaldas, horizontal. La valoración se registró a través de fotografías de los maniquís al finalizar la colocación de gomets para comprobar los resultados positivos y negativos.

La distribución de gomets de colores por concepto identificado es la siguiente:

\begin{tabular}{lllll}
\hline Delante & Detrás & Arriba & Abajo & Derecha \\
\end{tabular}

Tabla 2 - Distribución de colores por concepto a identificar en la prueba vivencial.

\subsubsection{Evaluación por dibujo.}

A los alumnos se les presentó un dibujo ya planteado que tenían que colorear siguiendo unas instrucciones. Se trata de un dibujo en el que podemos observar diferentes niños 
en distintas posiciones corporales en el que su eje corporal no es igual al eje normal de referencia (de pie). Estos niños están jugando a diferentes juegos y se observan objetos que los participantes deberán identificar y resolver de la mejor manera. Las indicaciones son las siguientes:

- María está jugando a la Rayuela: colorea de verde oscuro el arbusto que está a la DERECHA de María y de verde claro el arbusto que está a la IZQUIERDA de María.

- Paula no juego a la Rayuela, dibuja una pelota DELANTE de Paula.

- Dibuja un arcoíris DETRÁs de los arbustos.

- Pedro hace la voltereta, rodea de color rojo la parte de ARRIBA de su cuerpo y de color azul, la parte de ABAJO de su cuerpo.

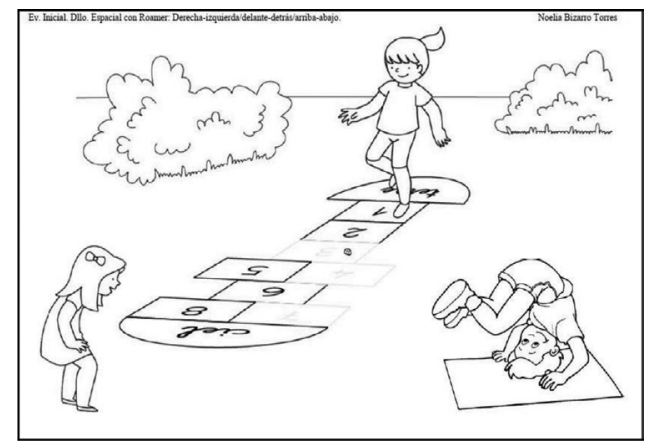

Figura 1.- Prueba de evaluación de dibujo.

\subsection{Procedimiento de análisis}

Posteriormente a la recogida de datos con los dos instrumentos empleados, se llevó a cabo el análisis de datos con el apoyo del software WebQDA. Primero, se introdujeron y se describieron los imágenes en las fuentes para después se codificaren en función de las categorías previamente definidas.

El procedimiento de análisis se llevó a cabo a través análisis cualitativo de las pruebas de identificación de conocimientos previos en la evaluación inicial y delas pruebas de evaluación del final del proceso, basado en las matrices de resultados generadas por webQDA.

\section{Actividades realizadas}

De acuerdo con la metodología planteada se desarrollaron las siguientes actividades:

1. Primera fase: Diseño de la investigación. En esta fase se planteó el problema que se pretendía resolver. Además se eligieron los conceptos espaciales que se pretendían trabajar y evaluar en los alumnos, se diseñó la investigación y también se programó el proyecto de intervención en el aula basado en la aplicación de actividades con robótica educativa y actividades corporales.

2. Segunda fase: Evaluación inicial ${ }^{1}$. Se llevó a cabo la prueba de evaluación vivencial (figura 2) y de dibujo (figura 3) justo antes de comenzar con el trabajo 
en el aula. Esta evaluación permitió conocer los conocimientos previos que tienen nuestros alumnos en referencia a los contenidos que vamos a trabajar.

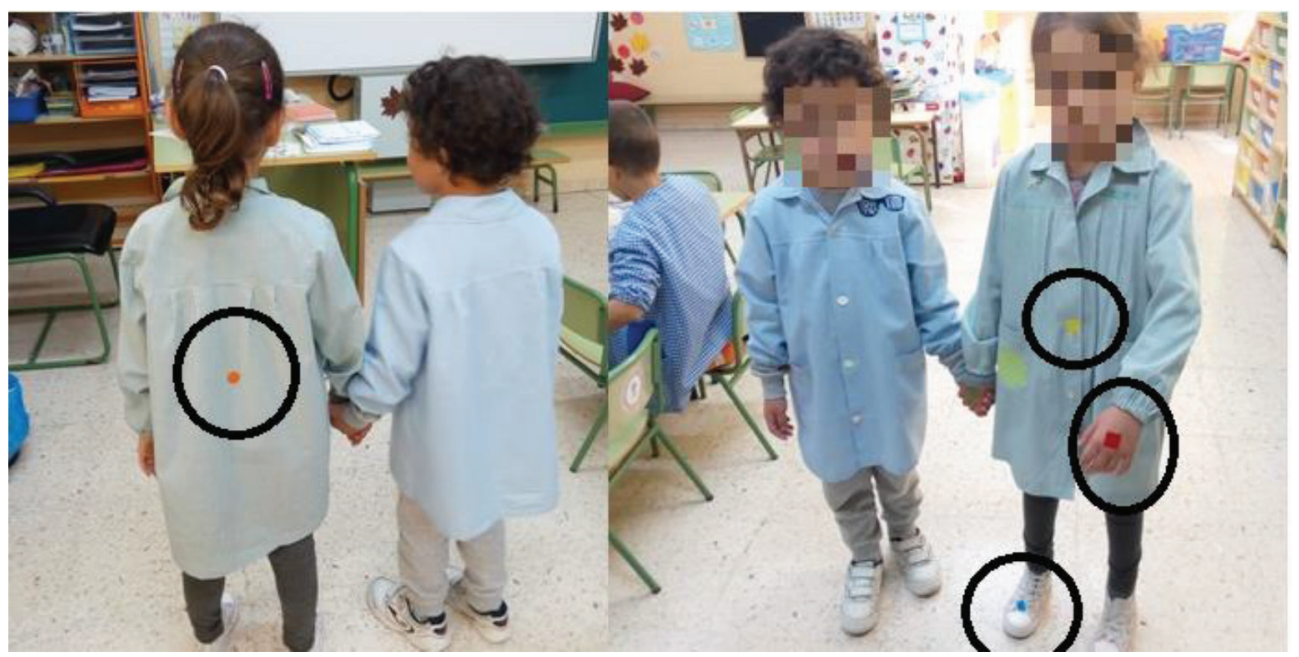

Figura 2.- Prueba vivencial de evaluación inicial.

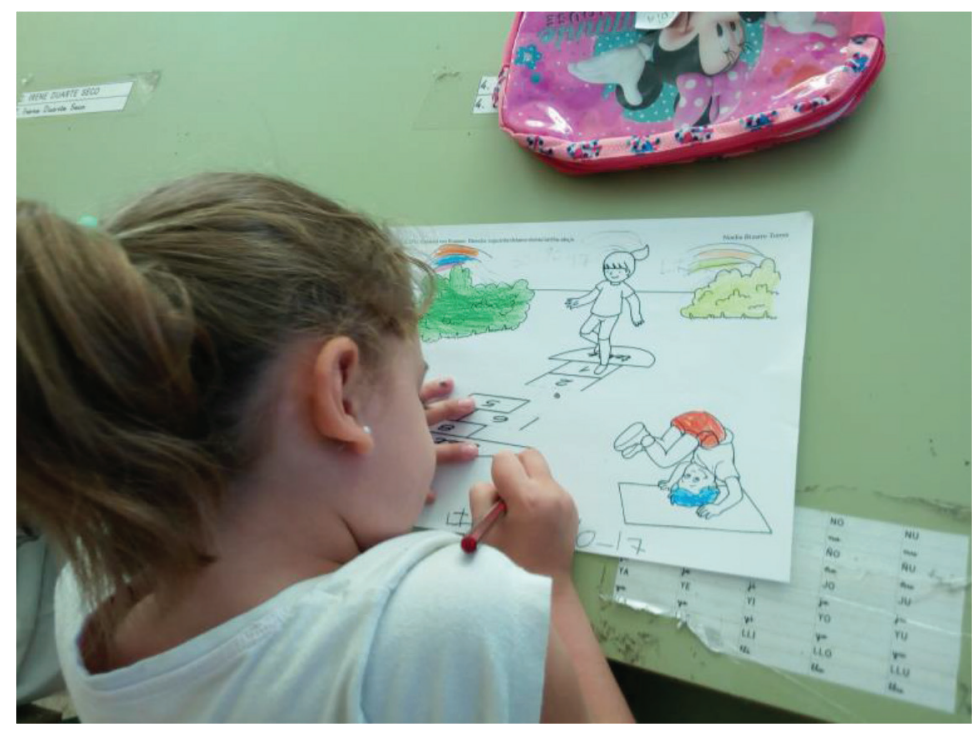

Figura 3.- Prueba de dibujo en evaluación inicial.

3. Tercera fase: Intervención en el aula. La intervención en el aula se llevó a cabo en el número de sesiones programadas inicialmente. Por un lado, se trabajó en el aula con robótica educativa contenidos de distintas áreas (Figura 4), 
dónde se presentaba el contenido de manera globalizada, aprovechando todas las potencialidades de esta herramienta. Y por otro lado, se han desarrollado actividades de psicomotricidad básicas (Figura 5) que complementan las actividades con robótica y que están relacionadas con los conceptos a trabajar.

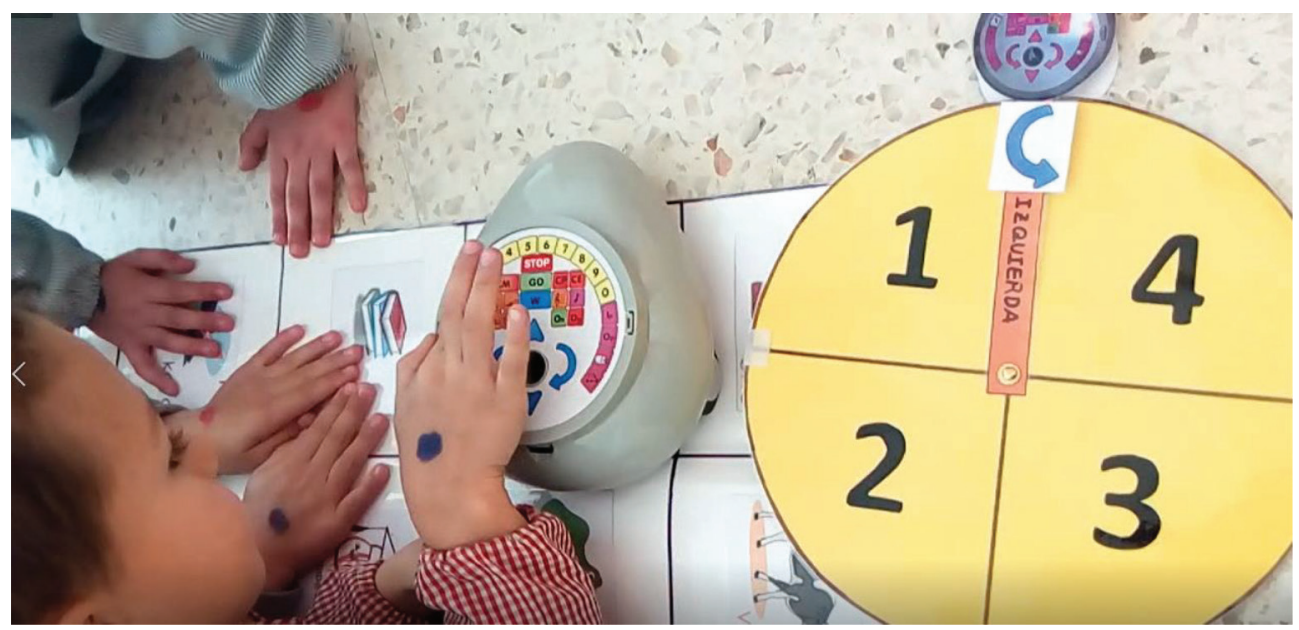

Figura 4 - Actividades en el aula con Robot Roamer.

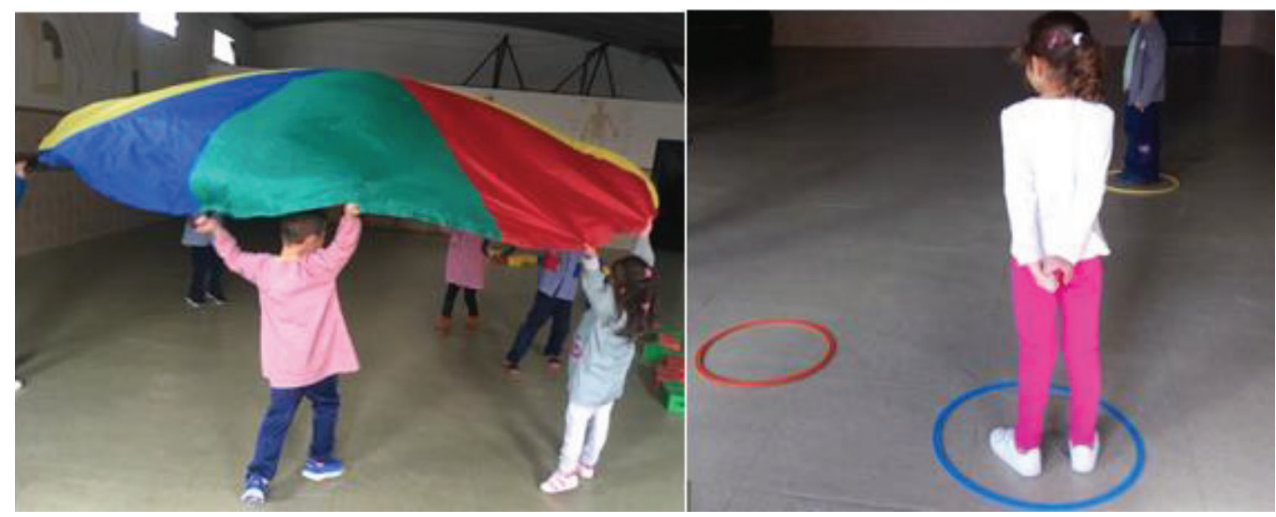

Figura 5 - Actividades en el aula de psicomotricidad.

4. Cuarta fase: Evaluación final ${ }^{2}$. Justamente después de la intervención en el aula con robótica educativa y actividades de psicomotricidad se repitió la evaluación vivencial y la prueba de dibujo. Se pretende observar si existen diferencias significativas con respecto a la evaluación inicial.

5. Quinta fase: Tratamiento de datos a través del software webQDA. En esta fase se llevó a cabo varias tareas secuenciadas:

- Transferencia de datos a webQDA: se incorporaron copias digitalizadas de las dos pruebas. En el caso de la prueba vivencial son fotografías y en el caso de la 
prueba de dibujo es el propio dibujo el que se incorporó a las fuentes internas del software. A través de webQDA se han señalado aquellos puntos referentes a los contenidos estudiados y se ha descrito su resolución.

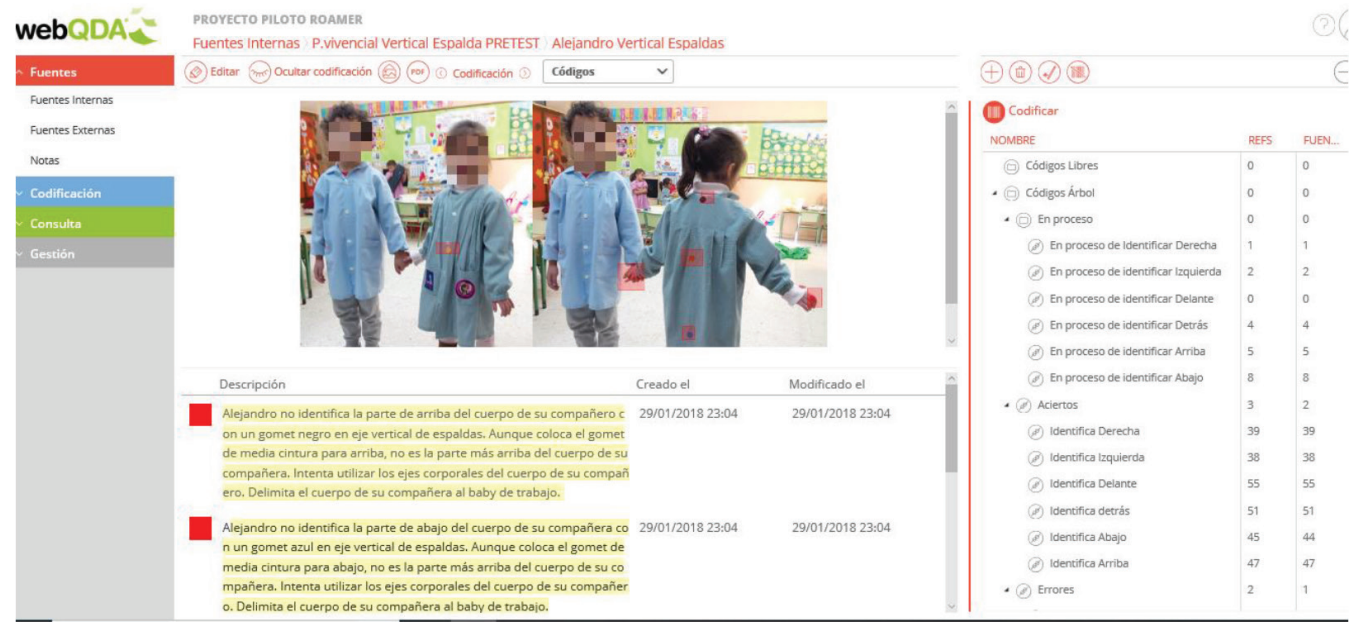

Figura 6 - Tratamiento de datos con el software webQDA.

- Creación del sistema de categorías: Posteriormente se creó un sistema de categorías para categorizar las respuestas de los alumnos. Las categorías se encuentran relacionadas con los 6 conceptos que se están evaluando y el proceso de adquisición de los mismos.

- Consulta de datos: Se llevó a cabo las consultas pertinentes para la obtención de resultados que se presentan en el siguiente apartado.

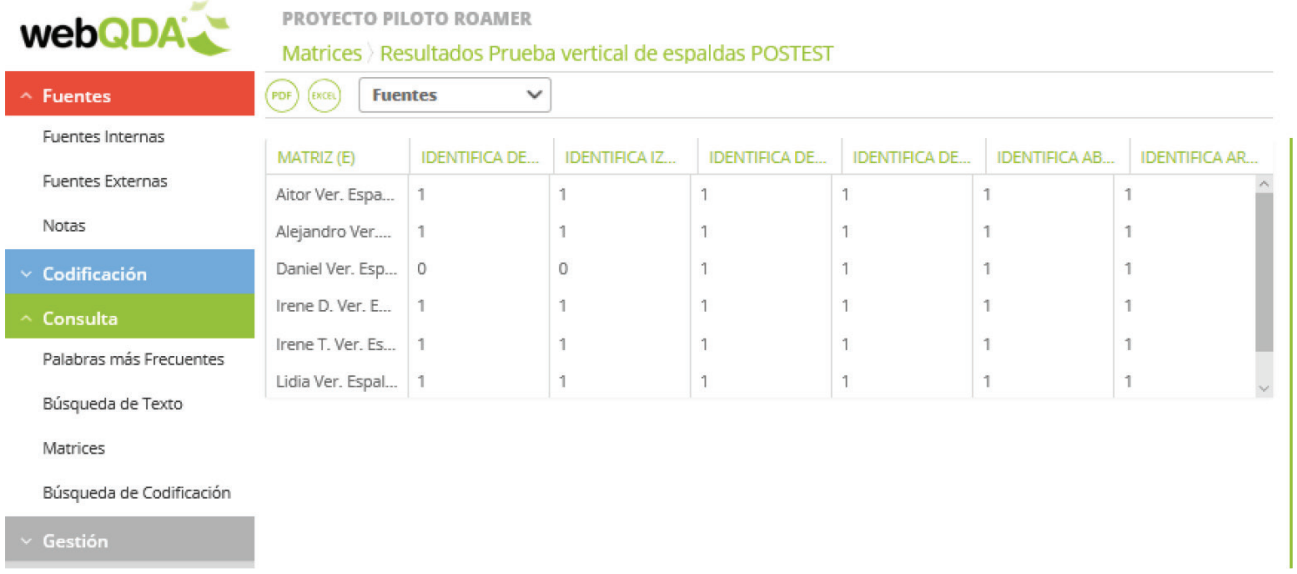

Figura 7 - Consulta de datos para la obtención de resultados con el software webQDA. 


\section{Resultados}

A continuación se exponen los resultados separados en las distintas pruebas que se llevaran a cabo teniendo en cuenta la comparativa de los momentos iniciales (pretest) y finales (postest) de su aplicación.

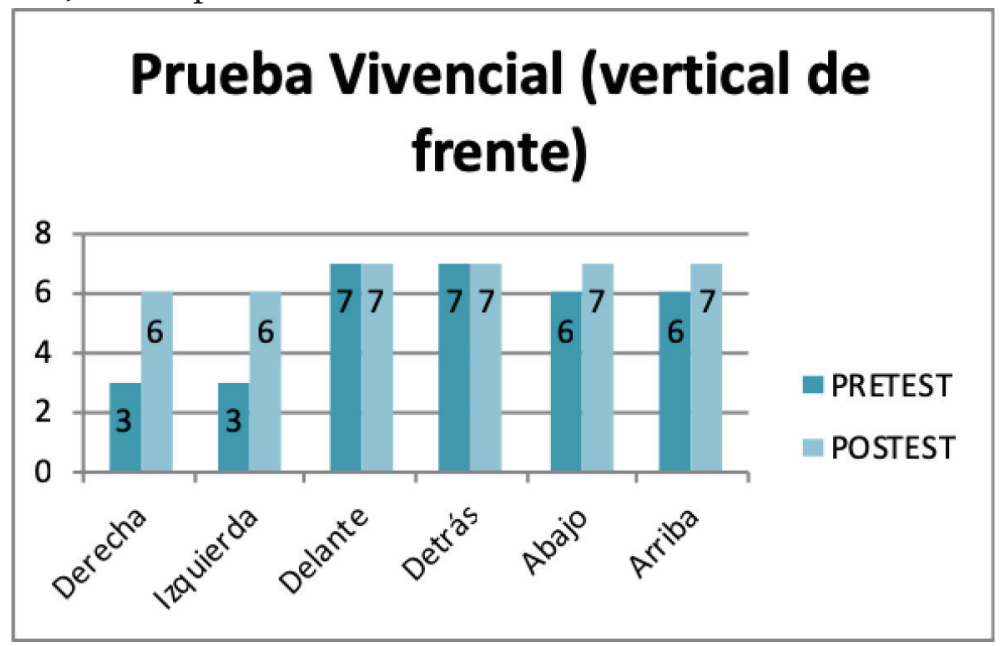

Figura 8 - Resultados de prueba vivencial de frente pretest y postest.

En la Figura 8 se pueden observar los resultados de la prueba vivencial de frente. En la prueba inicial, los alumnos mostraban mayores dificultades en la identificación de izquierda y derecha, pero en la prueba final se puede observar como en su mayoría han superado esas dificultades. Cabe destacar la dificultad de esta prueba ya que están identificando la izquierda y derecha en posición espejo, es decir en una posición contraria a la de su propio cuerpo.

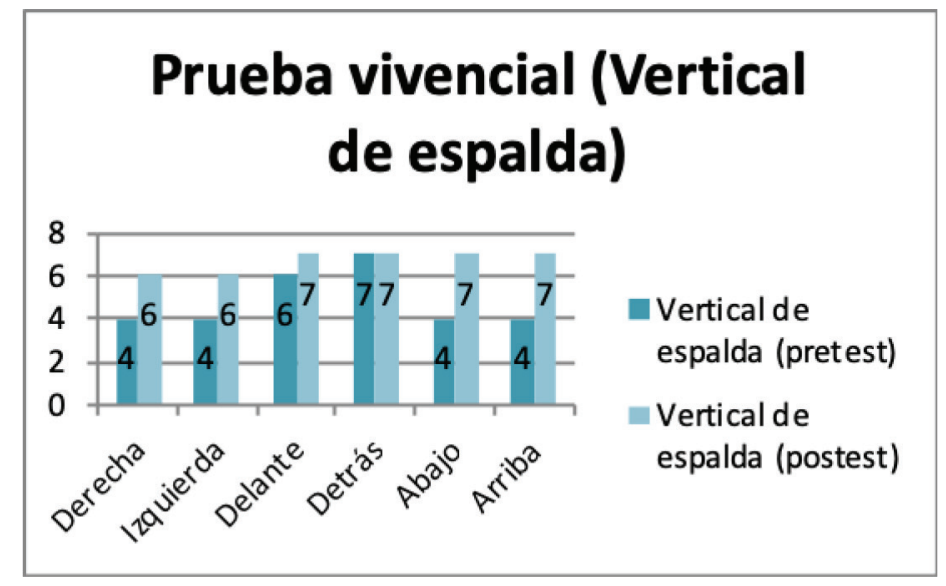

Figura 9 - Resultados de prueba vivencial de espalda pretest y postest. 
En la Figura 9, referente a la prueba vivencial vertical de espalda, los alumnos han mejorado la identificación de todos los conceptos excepto el concepto de izquierda y derecha, que sigue siendo el más complicado.

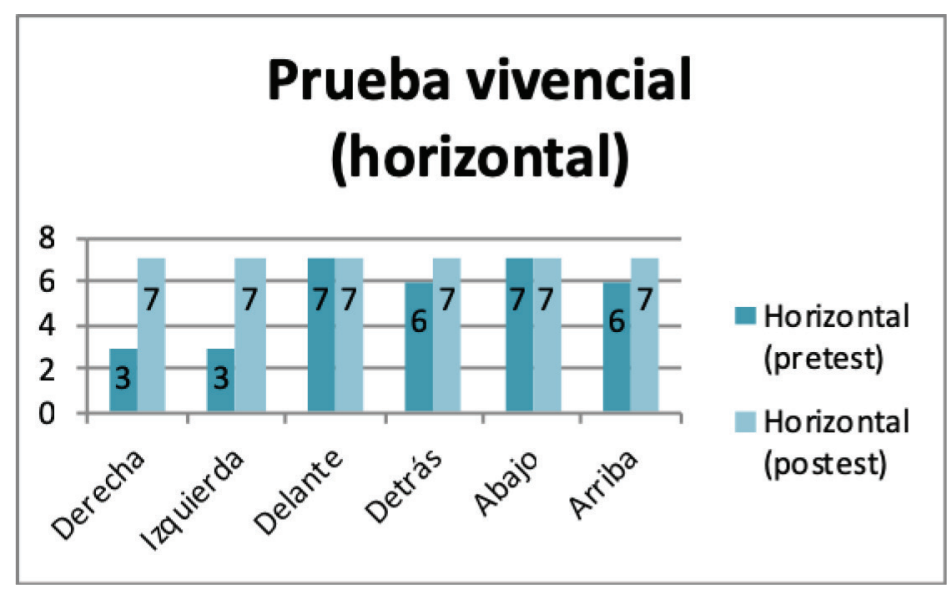

Figura 10 - Resultados de prueba vivencial horizontal pretest y postest.

En la Figura 10, relativa a la prueba vivencial horizontal, se observa cómo ha mejorado significativamente la correcta identificación de los conceptos que se piden a los alumnos. Cabe destacar que en posición horizontal si han sido capaz de identificar la derecha y la izquierda de su compañero.

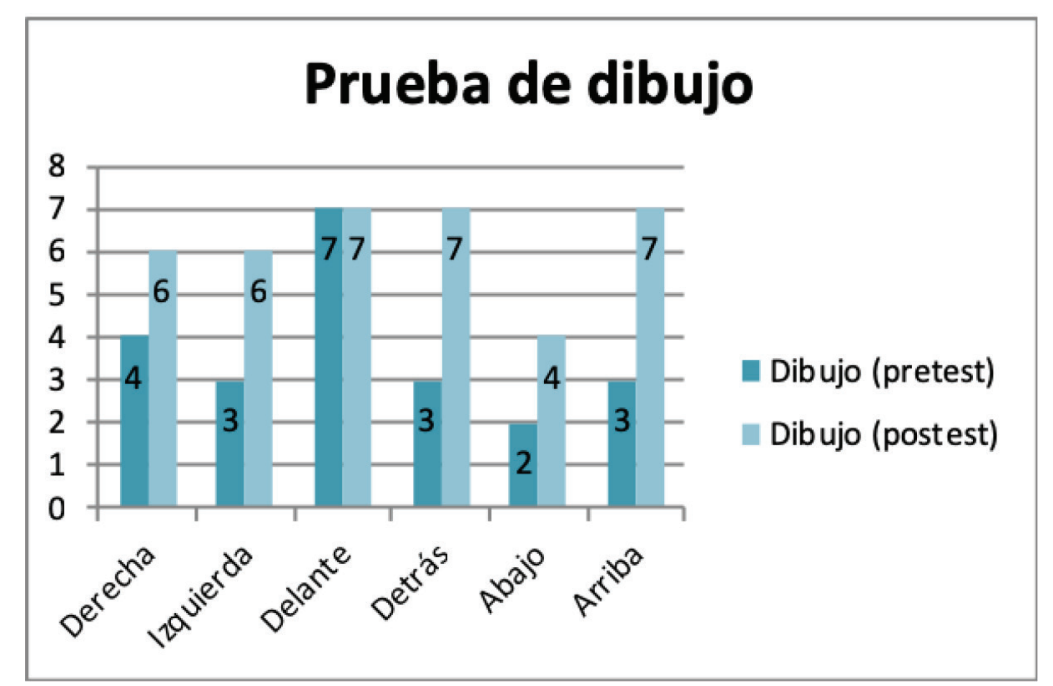

Figura 11 - Resultados de prueba de dibujo inicial y final. 
En la figura 11 se observan los resultados obtenidos de la prueba de dibujo. Los alumnos inicialmente presentaban problemas para la identificación de la derecha y la izquierda y también en arriba y abajo por la posición de la figura en la que tenían que identificar estos últimos conceptos (Pedro de la prueba de dibujo). Sin embargo, en la evaluación final se ha podido observar cómo los alumnos mejoran la identificación de todos estos conceptos, aunque siguen teniendo dificultades para identificar el concepto abajo.

\section{Conclusiones y sugerencias}

El trabajo con robótica en el aula mejora la adquisición de conceptos espaciales básicos en alumnos de educación infantil y ayuda al niño a adaptarse a las características de pensamiento irreversible que defiende Piaget en Feldman (2007). Sin embargo, se debe tener presente que los conceptos de derecha e izquierda son los más complicados para estos alumnos, ya que en todas las pruebas realizadas se observa las dificultades que tiene el alumno para su identificación. Por ello se propone incidir más en las actividades de la intervención en el aula sobre estos conceptos (Bizarro, Luengo y Carvalho, 2018).

En referencia a las cuestiones específicas planteadas, se ha llevado a cabo la observación, análisis y conclusiones sobre las diferencias entre las pruebas aplicadas en los dos momentos de recogida de datos. Además también se ha diseñado un proyecto de intervención educativa con materiales manipulativos y actividades atractivas para el alumnado referente a la robótica educativa en el aula y psicomotricidad, respetando el principio de globalización, motivación y juego. Los alumnos han trabajado favorablemente en el aula con el material elegido. Se mostraban motivados y participativos en todas las actividades, por ello el trabajo con robótica en el aula es muy positivo, ya que mantiene motivado al alumno y favorece la aplicación de estrategias de resolución de problema. A través de este proceso se ha trabajado el desarrollo del "Computational Thinking" (Wing, 2006).

El equipo de investigación ha valorado muy positivamente el aporte de la metodología cualitativa y el enfoque metodológico de la misma, considerando también que el uso del programa de análisis cualitativo webQDA facilitó extraordinariamente la organización y estructuración de las fuentes extraídas de las pruebas de evaluación, la codificación e interpretación de los datos, así como la consecución de conclusiones.

Como perspectiva de desarrollo futuro plantease la comparación de estas dos pruebas de evaluación con otras que permitirán triangular la obtención y análisis de datos y resultados a partir de diferentes instrumentos. Este estudio será replicado aplicando un test estándar que evalúe los conceptos trabajados y utilizando la técnica de Redes Asociativas Pathfinder (Casas, Luengo y Godinho, 2011) para analizar la estructura cognitiva de los alumnos, antes y después de las intervenciones en el aula. En concreto, se pretende comprobar si los resultados de la prueba estándar y de la prueba de Redes Associativas Pathinder (entre el grupo experimental y el grupo de controlo antes y después de la intervención en el aula con robótica educativa) son significativos y se complementan o presentan resultados dispares con los registros de observación realizados durante o estudo piloto.

En base a las conclusiones alcanzadas y las limitaciones propuestas se sugiere la réplica del estudio con una muestra más amplia y observando diferencias frente a la utilización de una metodología tradicional en el aula, es decir, sin la utilización de actividades robótica por un grupo de control. 


\section{Referencias}

Alsina, A. (2012). Hacia un enfoque globalizado de la educación matemáticas en las primeras edades. Números: Revista de Didáctica de las Matemáticas, 80, 7-24.

Bizarro, N., Luengo, R., \& Carvalho, J. L. (2018). Desarrollo de nociones espaciales básicas a través del trabajo con Robótica Educativa en el Aula de Educación Infantil y análisis de datos cualitativos con Software webQDA. En: Actas del CIAIQ 2018 Congreso IberoAmerciano de Investigación Cualitativa. Fortaleza: UNIFOR.

Blikstein, P. (2013). Seymour Papert's Legacy: Thinking About Learning, and Learning About Thinking. Retreived from: https://ttl.stanford.edu/content/seymourpapert-s-legacy-thinking-about-learning-and-learning-about-thinking

Casas, L., Luengo, R. \& Godinho, V. (2011). Software MICROGOLUCA: Knowledge Representatión in Mental Calculatión. Us-China Education Reviex, 1(4), 592-600.

Costa, A., Faria, B., \& Reis, L. (2016). Investigação Qualitativa Através da Utilização de Software: Workflows Metodológicos. RISTI - Revista Ibérica de Sistemas e Tecnologias de Informação, (19), IX-XII.

Costa, A., \& Reis, L. (2017). Vantagens e desvantagens do uso de software na análise de dados qualitativos. RISTI - Revista Ibérica de Sistemas e Tecnologias de Informação, (23), IX-XIII.

Decreto 4/2008 de 11 de Enero por el que se establece el currículo de Educación Infantil para la Comunidad Autónoma de Extremadura.

Feldman, R.S. (2007). Desarrollo psicológico a través de la vida. México: Pearson Educación.

Ferrão, S., Marques, R., \& Moreia, A. (2013). WebQDA na análise qualitativa de interações no contexto de uma oficina de formação de professores. Indagatio Didactica, 5(2), 110-121.

López-Pastor, V.M., Pérez-Pueyo, A, et al. (2017). Evaluación formativa y compartida en Educación: Experiencias de éxito en todas las etapas educativas. Universidad de León. Servicio de publicaciones

Neri de Souza, F., Costa, A.P., Moreira, A. \& Neri de Souza, D. (2013). WebQDA - Manual do Utilizador. Universidad de Aveiro, Portugal: Esfera Crítica.

Rocha, Á. (2012). Framework for a Global Quality Evaluation of a Website. Online Information Review, 36(3), 374-382. doi:10.1108/14684521211241404

Valencia, M. (2016). Cuando los niños aprenden robótica y programación. El Español. Recuperado de: https://www.elespanol.com/ciencia/ tecnologia/20160301/106239504_o.html

Valverde, J., Fernández, M.R., \& Garrido, M.C. (2015). El pensamiento computacional y las nuevas ecologías del aprendizaje. Revista de Educación a Distancia, 46(3)

Wing, J. M. (2006). Computational Thinking. Communications of the ACM, 49(3), 33-35. 